\title{
Diffraction \& Forward Physics in CMS: results and perspectives
}

\author{
Patricia Rebello Teles ${ }^{1, a}$ on behalf of the CMS Collaboration \\ ${ }^{1}$ Centro Brasileiro de Pesquisas Físicas - CBPF, Rio de Janeiro-RJ, 22290-180, Brazil
}

\begin{abstract}
In this plenary talk given at ISMD2014 the interesting CMS analyses on diffractive proton-proton cross section and Mueller-Navelet dijet azimuthal decorrelations performed at $\sqrt{s}=7 \mathrm{TeV}$ were presented.
\end{abstract}

\section{The CMS Detector: Central \& Forward regions}

A detailed description of the CMS detector can be found in Ref. [1]. According Fig. 1, in the central part of the apparatus are located the electromagnetic and hadronic calorimeters (ECAL and HCAL, respectively) as well as the forward component of the hadron calorimeter, $\mathrm{HF}$ (Hadronic Forward), covering the $2.9<|\eta|<5.2$ region. The very forward angles are covered at one end of CMS (the $6.6<|\eta|<5.2$ region) by the CASTOR calorimeter. In addition, two elements of the CMS monitoring system, the Beam Scintillator Counters (BSC) and the Beam Pickup Timing eXperiment (BPTX) devices, are used to trigger the CMS readout. In the same figure the joint set of CMSTOTEM near-beam detectors is shown as well.

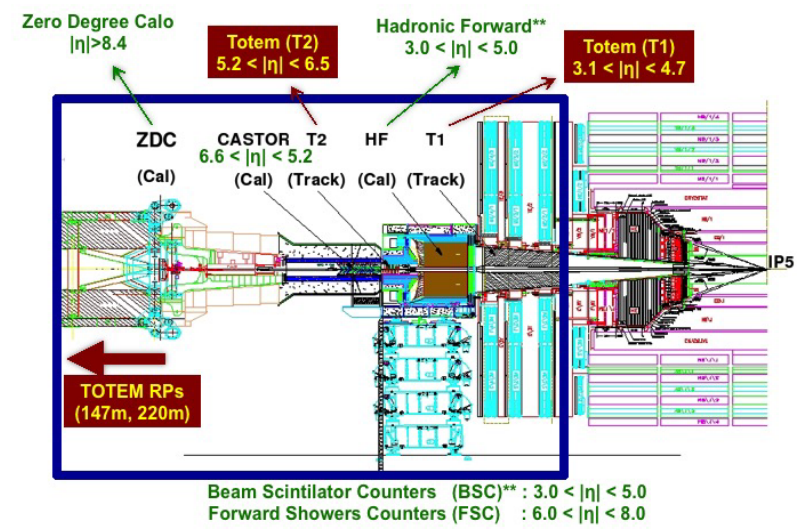

Figure 1. Forward region - The CMS and TOTEM Collaborations (both sides)

\section{Diffraction at CMS: the pp diffraction dissociation cross section at $\sqrt{s}=7 \mathrm{TeV}$}

The composition of the total pp cross section is

$$
\sigma_{t o t}=\sigma_{E L}+\sigma_{S D}+\sigma_{D D}+\sigma_{C D}+\sigma_{N D}
$$

where "SD" means Single-Diffractive, "DD" means Double Diffractive, "CD" means Central Diffractive and "ND" means Non-Diffractive, all belonging to the inelastic cross section $\sigma_{I N E L}$.

At LHC the elastic cross section, $\sigma_{E L} \approx 25 \mathrm{mb}$, is about $20 \%$ of the $\sigma_{\text {tot }}[2]$ and the inelastic, $\sigma_{I N E L} \approx 69 \mathrm{mb}$, is responsible for about $80 \%$ of $\sigma_{t o t}$ [3].

Inside the inelastic part the diffractive processes are characterized by large rapidity gaps (LRG), $\Delta \eta=\eta_{\text {max }}-$ $\eta_{\min }$ where $\eta=(1 / 2) \ln \left[\left(E+p_{z}\right) /\left(E-p_{z}\right)\right]$ according to Fig. 2.

Moreover, hadronic interactions with LRG are mediated by Pomeron exchange, based on the trajectory of Regge theory $\alpha(t)=1+\epsilon+\alpha^{\prime} t$, with $t=\left(p_{1}-p_{2}\right)^{2}$.

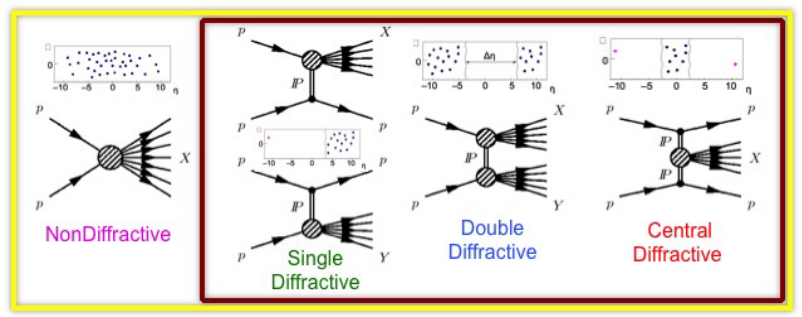

Figure 2. Diffractive and non-diffractive processes in pp collisions.

The first CMS analysis presented in this talk [4] has used the PYTHIA8-MBR (Minimum Bias Rockefeller) [5] and PYTHIA8-4C (with diffraction from Schuler \& Sjostrand) [6] for comparison, with $\alpha^{\prime}=0.25 \mathrm{GeV}^{-2}$. The data were collected in 2010 using a low pileup scenario which is the most suitable for diffractive event selection using a LRG signature.

a e-mail: patricia.rebello.teles@ cern.ch 
Signal in both BPTX (Beam Pick-up Timing Experiment) detectors and activity in any of the BSC $(3.2<|\eta|<$ 4.7) were required for the presence of two bunches along with minimal activity in the central CMS detector (Minimum Bias trigger). In addition, Single Diffractive (SD) and Double Diffractive (DD) events were separated with CASTOR $(6.6<|\eta|<5.2)$.

To accept low to moderate diffractive masses $(12<$ $\mathrm{M}_{X}<100 \mathrm{GeV}$ ), no vertex was required. The diffractive offline selection was based on LRG within $|\eta|<4.7$ (HF was not used, therefore limiting central CMS detector coverage), the proton momentum loss $\xi$ was reconstructed from particles in $|\eta|<4.7$ according expressions ${ }^{1}$

$$
\xi^{ \pm}=\frac{\Sigma\left(E^{i} \mp p_{z}^{i}\right)}{\sqrt{s}} \quad \xi=\frac{\mathrm{M}_{X}^{2}}{s},
$$

where $\mathrm{M}_{X}$ is the mas of the diffractive system.

Three experimental topologies based on the position of the LRG were analysed according to Fig. 3, as well as the distributions for the Mininum Bias data samples, following the aforementioned experimental topologies, compared with PYTHIA8-MBR predictions. The cuts to select the samples for SD1, SD2 and DD topologies with a central LRG signature were $\eta_{\max }<1, \eta_{\min }>-1$ and $\Delta \eta_{0}>3$, respectively, where $\eta_{\max (\min )}$ is the highest (lowest) $\eta$ of a particle candidate within $|\eta|<4.7$.

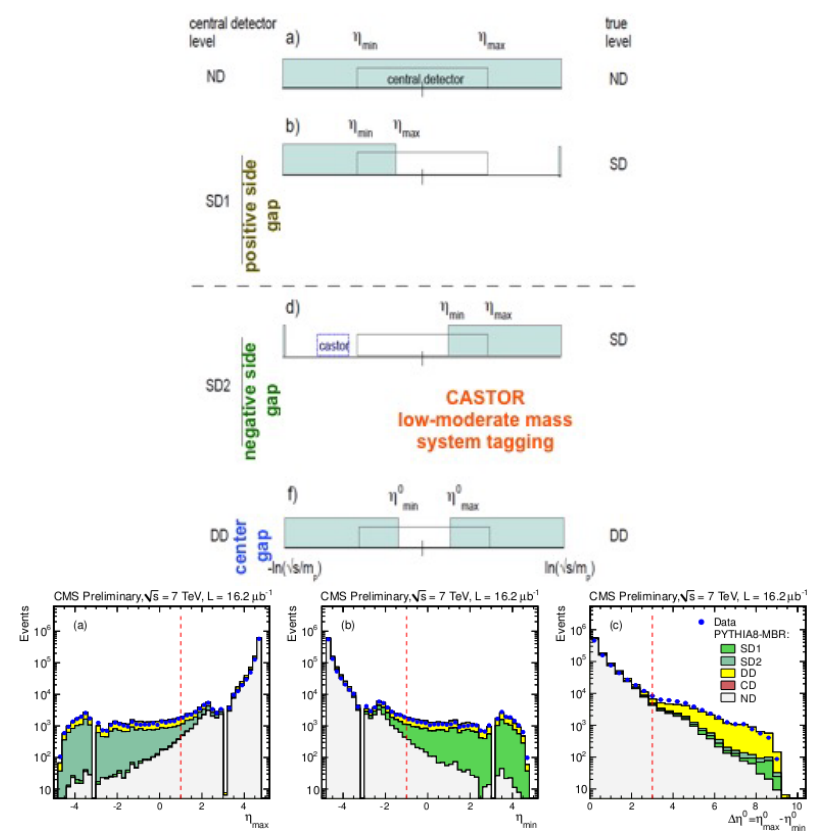

Figure 3. In the top panel we show the experimental topologies based on the position of the LRG, where $|\eta|<\ln \left(\sqrt{s} / m_{p}\right)=$ 8.92. In the bottom, the distributions for the Minimum Bias data samples compared with PYTHIA8-MBR predictions according the experimental topologies are shown.

In Fig. 4 the SD and DD contribution from the SD2 event sample (three samples tagged according CASTOR) are best described by Pythia8-MBR tune.

\footnotetext{
${ }^{1}$ Detector level; " $\mathrm{i}$ " runs over all Particle Flow objects measured in the central detector; dissociated system occurs on the $\pm \mathrm{Z}$ side of the detector.
}

One can see the SD and DD contributions from SD2 event sample in the top of the Fig. 5. Comparison with PYTHIA8-MBR, PYTHIA8-4C, and PYTHIA6 [7] MC simulations were done with two values of Pomeron intercept in the MBR model. It is shown that PYTHIA8$4 \mathrm{C}$ does not reproduce the falling behavior of SD and in the $-5.5<\log _{10} \xi<-2.5$ region the total SD cross section reaches $\sigma_{\text {Total }}^{S D}=4.27 \pm 0.04$ (stat. ${ }_{-0.58}^{+0.65}$ (syst.) mb.

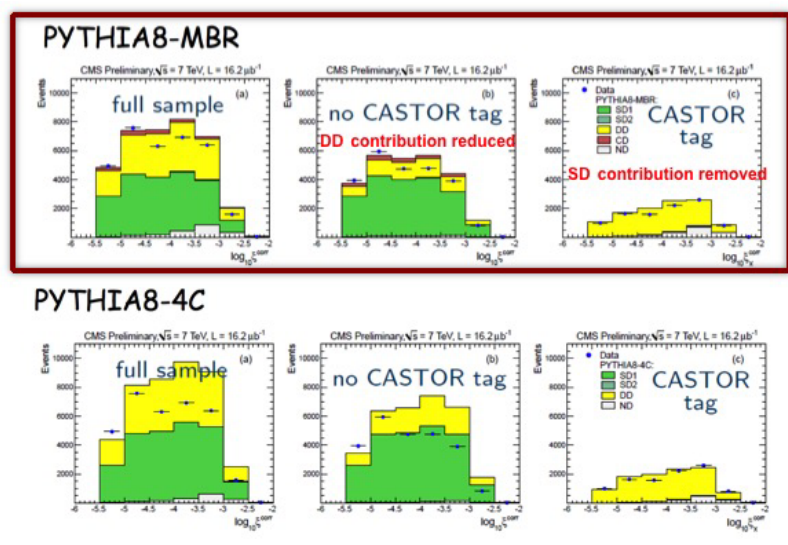

Figure 4. SD and DD contribution from SD2 event sample, best delineated by Pythia8-MBR tuning.

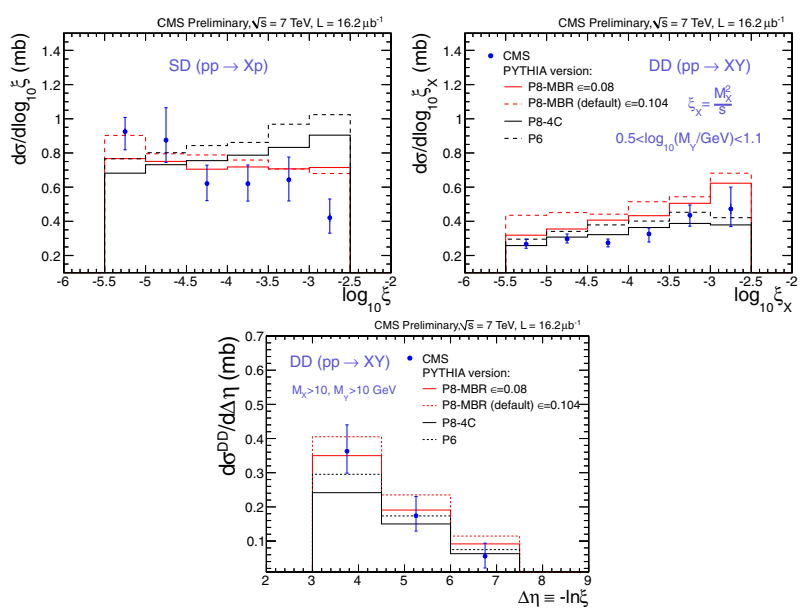

Figure 5. In the top panel, the SD and DD contributions from SD2 event sample. In the bottom, DD contribution from DD event sample.

In addition, the DD contribution from DD event sample is shown in the right side of the Fig. 5. Over the region $\Delta \eta>3, M_{X}>10 \mathrm{GeV}$ and $M_{Y}>10 \mathrm{GeV}$, the cross section value approaches $\sigma^{D D}=0.93 \pm 0.01$ (stat. $)_{-0.22}^{+0.26}$ (syst.) $\mathrm{mb}$.

An alternative approach to the study of diffractive events is to measure the differential cross section of the forward rapidity gap " $\Delta \eta^{F}$. In Fig. 6 we can notice the exponential falling of the ND contribution and the diffractive plateau at $\Delta \eta^{F}>3$ with mixtures of SD and DD events for all stable final-state particles with $\mathrm{pT}>200 \mathrm{MeV}$ in $|\eta|<4$.7. A good description using PYTHIA8-MBR with 

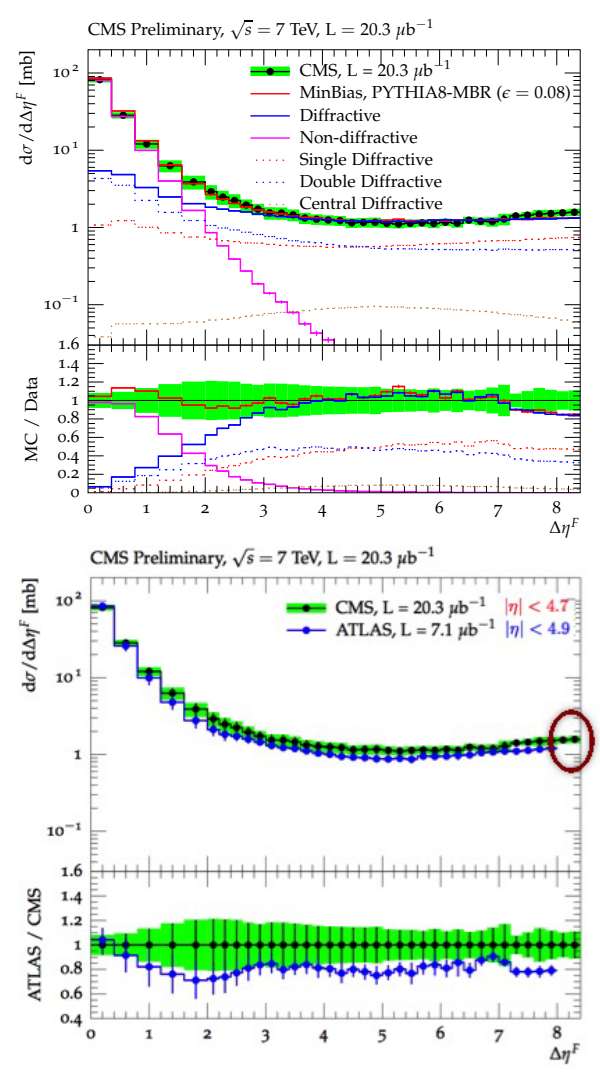

Figure 6. In the top panel one has the differential cross section in the forward rapidity gap $\left(\Delta \eta^{F}\right)$. In the bottom panel is shown the CMS and ATLAS comparison for the differential cross section of the $\Delta \eta^{F}$. The CMS result extends the ATLAS measurement by 0.4 unit of gap size.

$\epsilon=0.08$ intercept is reported. Moreover, the CMS result extends the ATLAS measurement by 0.4 unit of gap size.

\section{Forward Jets at CMS: the azimuthal angle decorrelations of Mueller-Navelet jets at $\sqrt{s}=7 \mathrm{TeV}$}

In the hard processes regime $\left(\sqrt{s} \geq \mathrm{pT} \gg \Lambda_{Q C D}\right)$, data are well-described by perturbative QCD within the DGLAP evolution equation (mainly pT ordered). On the other hand, for the semi-hard processes $(\sqrt{s} \gg \mathrm{pT} \gg$ $\left.\Delta_{Q C D}\right)$ the asymptotic region $(\sqrt{s} \rightarrow \infty)$ is described by the BFKL approach, that is the number of emitted partons increases with an increasing rapidity interval $\Delta \eta=\left|\eta_{1}-\eta_{2}\right|$ between the Muller-Navelet (MN) jets and, hence, the MN jets are no longer back-to-back $(\Delta \phi=\pi)$ in azimuthal direction, leading to a decorrelation. The observation of such decorrelations, see Fig. 7 expressions, indicates the presence of BFKL contributions [8].

The measurement conditions in the second CMS analysis of this talk [8] were $\Delta \eta_{M N J e t s}<9.4$, two jets with pT $>35 \mathrm{GeV}$ and $|\eta|<4.7$ using the anti-kT algorithm with jet size $\mathrm{R}=0.5$. Several observables, like the azimuthal, $\Delta \phi$, distribution as a function of $\Delta \eta$; the average cosines $C_{n}=\langle\cos (n(\pi-\Delta \phi))\rangle$ with $\mathrm{n}=1,2,3$; and the ratios $\mathrm{C} 2 / \mathrm{C} 1$ and $\mathrm{C} 3 / \mathrm{C} 2$ were analysed.

$$
\begin{gathered}
\frac{1}{\sigma} \frac{d \sigma}{d(\Delta \phi)}\left(\Delta y, p_{\operatorname{Tmin}}\right)=\frac{1}{2 \pi}\left[1+2 \sum_{n=1}^{\infty} C_{n}\left(\Delta y, p_{\operatorname{Tmin}}\right) \cdot \cos (n(\pi-\Delta \phi))\right] \\
C_{n}\left(\Delta y, p_{\operatorname{Tmin}}\right)=\langle\cos (n(\pi-\Delta \phi))\rangle<1
\end{gathered}
$$

Figure 7. Azimuthal angle distribution and correlation coefficients expressions.

In Fig. 8 the $\Delta \phi$ distributions in two rapidity intervals $(\Delta \eta<3.0$ and $6.0<\Delta \eta<9.4)$ are shown. We can notice azimuthal decorrelation raising with increasing $|\Delta \eta|$ as expected. Concerning the MC generators, the HERWIG++ shows the best description in all bins, the PYTHIA, SHERPA and CASCADE present too large decorrelation.
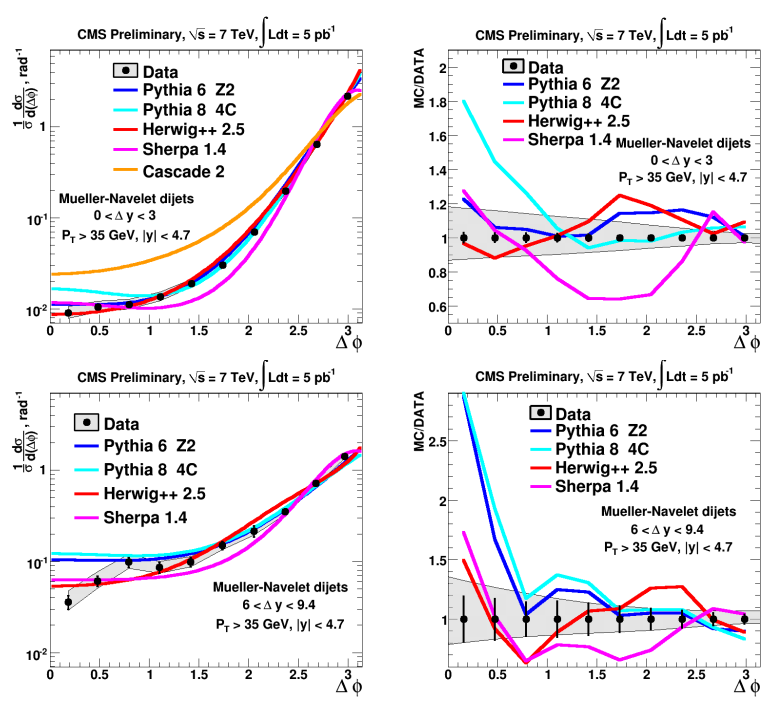

Figure 8. The $\Delta \phi$ distributions in three rapidity intervals $(\Delta \eta<$ 3.0 and $6.0<\Delta \eta<9.4$ )

In the comparison plots for the average cosine $C_{n}$ of Fig.9 the measured average cosines decrease with increasing $\Delta \eta$ indicating increasing decorrelation of jets in azimuthal angle. The MC generators PYTHIA and HERWIG++ agree mostly with the data, while SHERPA, CASCADE and NLL BFKL do not, being either above or below them ${ }^{2}$. It is important to notice that, at large values of $\Delta \eta$, additional emissions are becoming kinematically suppressed due to energy-momentum conservation near the phase-space boundary. Thus, coplanarity of MN jets results in an increase of average cosines.

The azimuthal angle decorrelation depends also on multiple particle interactions (MPI), producing additional jets not correlated with those from the primary interaction. The Figs. 10 and 12 show the polar angle ordering (AO) effects in the parton showering and multiparton interactions (MPI), and indeed the average cosines $C_{n}$ are very sensitive. A good description of the data requires polar angle ordering in parton showering.

\footnotetext{
${ }^{2}$ The newer NLL BFKL claimed to be better according to http://arxiv.org/abs/arXiv:1309.3229
} 

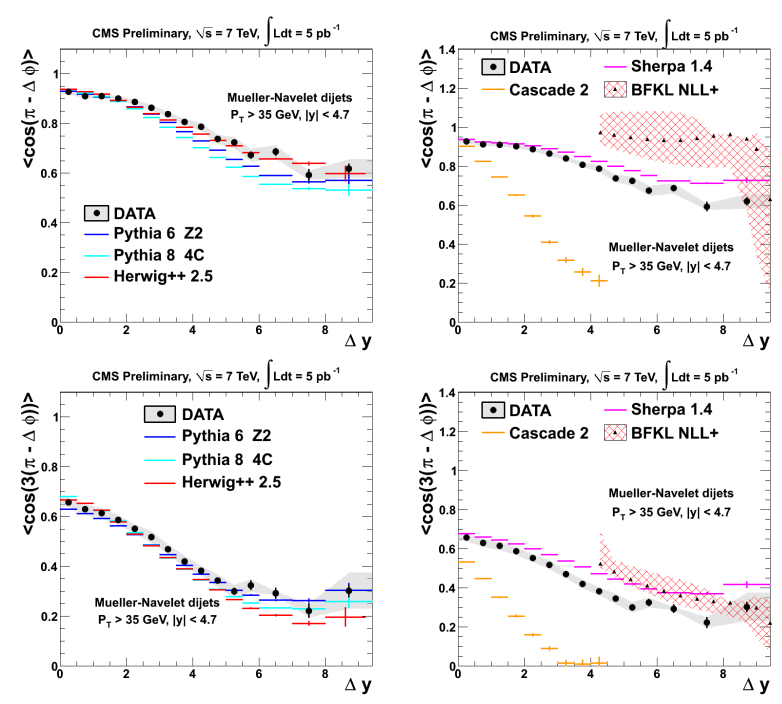

Figure 9. Comparison plots for the average $\operatorname{cosine} C_{n}$. The measured average cosines decrease with increasing $\Delta \eta$ indicating increasing decorrelation of jets in azimuthal angle.
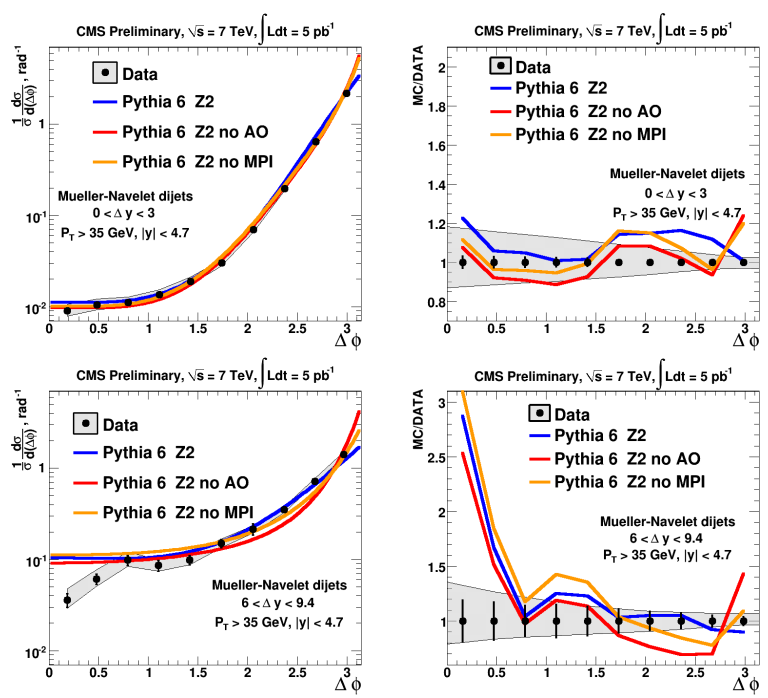

Figure 10. Distributions of the azimuthal angle showing the polar angle ordering $(\mathrm{AO})$ effects in the parton showering and multiparton interactions (MPI).
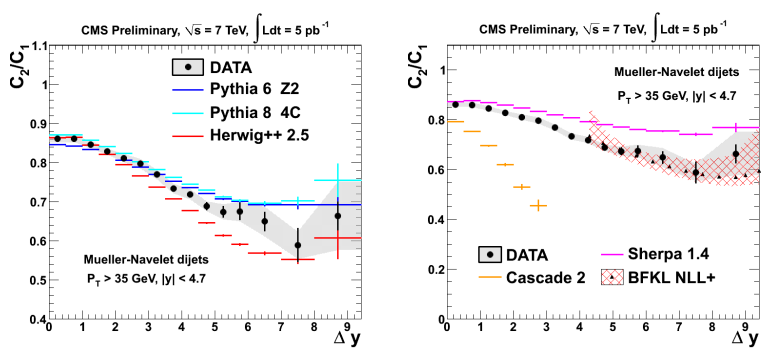

Figure 11. The measured ratios of $C_{n}$ against $\Delta \mathrm{y}$.
The ratios of $C_{n}$ are expected to be more sensitive to BFKL effects (See Fig. 11). PYTHIA and HERWIG++ show good agreement at low $\Delta \eta$, but discrepancies in the large $\Delta \eta$ region. On the other hand SHERPA is above the data while CASCADE is far below the data. NLL BFKL calculation describes the ratios quite well, especially the $C_{2} / C_{1}$ one.

\section{Summary}

Two interesting CMS results related with inclusive Single Diffractive and Double Diffractive cross section measurement at $7 \mathrm{TeV}$ and BFKL effects through azimuthal decorrelations in $\mathrm{MN}$ jets were presented.

The forward and diffractive physics show as a perfect testing ground for models and theories [10].

We notice the importance of Monte Carlo generators comparisons and tuning for better data matching. In addition, several $8 \mathrm{TeV}$ analyses, with 2012 data and integrated luminosity $\approx 20 \mathrm{fb}^{-1}$ are in progress.

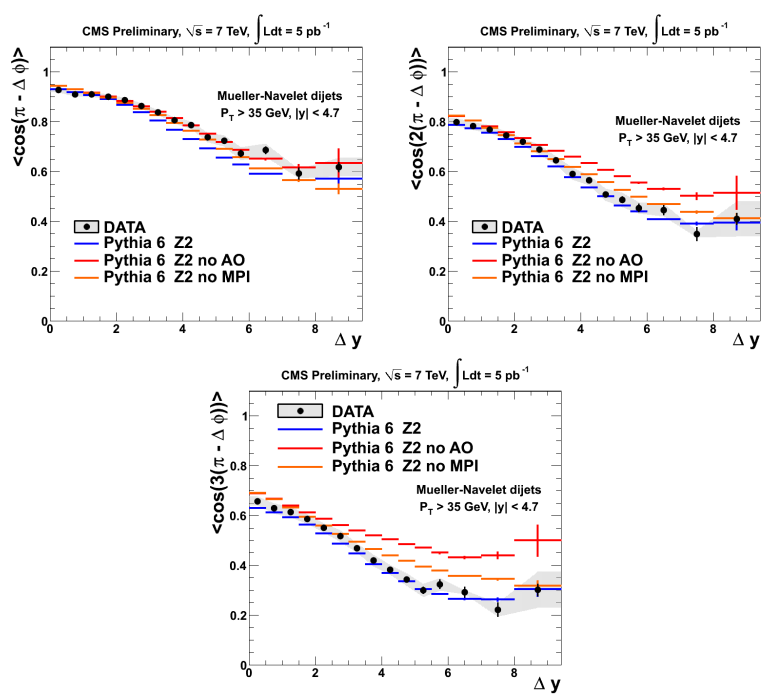

Figure 12. The measured average cosines $C_{n}$ with $\mathrm{AO}$ in the parton showering and MPI effects versus $\Delta y$.

\section{References}

[1] CMS Collaboration, JINST 03 (2008) S08004.

[2] TOTEM Collaboration, Europhys.Lett. 95 (2011) 41001.

[3] CMS Collaboration, Phys.Lett. B722 (2013) 5-27.

[4] S. Chatrchyan et al. [CMS Collaboration], CMS-PAS-FSQ-12005, CERN, Geneva (2013).

[5] R. Ciesielski and K. Gouliano, arXiv:1205.1446.

[6] T. Sjostrand et al., Comput.Phys.Commun. 178, 852 (2008).

[7] T. Sjostrand et al., JHEP 05, 026 (2006).

[8] S. Chatrchyan et al. [CMS Collaboration], CMS-PAS-FSQ-12002, CERN, Geneva (2013).

[9] S. Chatrchyan et al. [CMS Collaboration], JHEP 1307, 116 (2013) [arXiv:1305.5596 [hep-ex]].

[10] https://twiki.cern.ch/twiki/bin/view/CMSPublic/PhysicsResultsFSQ 